\title{
Investigating Plantar Fasciitis
}

\author{
by Valerie A.J. Potter, MBChB, BSc (Hons), $\mathrm{MSc}^{1 \otimes}$
}

The Foot and Ankle Online Journal 2 (11): 4

Plantar fasciitis is a condition caused by inflammation of the plantar aponeurosis and is a common cause of heel pain. The diagnosis is clinical and investigations are generally not required. However, in certain cases such as an atypical presentation or where there is no response to treatment, the clinician may consider further investigation. This review article briefly introduces the condition and goes on to discuss the possible options for investigation. This includes evaluation and usefulness of such tests as blood evaluation, radiographs, ultrasound, bone scintigraphy, magnetic resonance imaging (MRI) and nerve conduction studies.

Key words: Plantar fasciitis, investigations, heel pain, MRI, ultrasound, bone scintigraphy, nerve conduction.

Accepted: October, $2009 \quad$ Published: November, 2009

This is an Open Access article distributed under the terms of the Creative Commons Attribution License. It permits unrestricted use, distribution, and reproduction in any medium, provided the original work is properly cited. (The Foot and Ankle Online Journal (www.faoj.org)

Anatomy and etiology of plantar fasciitis

$\mathrm{T}$ he plantar fascia of the foot refers to the deep fascia of the sole. It arises posteriorly from the calcaneus and divides into bands which divide to enclose tendons. The thick central part of this fascia forms the plantar aponeurosis which is arranged into bands of fibrous connective tissue. The plantar fascia has a number of roles, which include holding parts of the foot together, protecting the plantar surface of the foot from trauma and helping to support the foot's longitudinal arch. 'Plantar fasciitis' refers to inflammation of the plantar aponeurosis. This is a relatively common clinical condition and cause of heel pain resulting from a degenerative process. The exact mechanism is poorly understood but thought to be multifactorial. Mechanical overload is believed to play a key role. ${ }^{1}$ Risk factors include age, obesity, biomechanical abnormalities of the foot and particular occupation- related or recreational activities such as aerobics and running particularly in flat or worn shoes.

Address correspondence to: East Surrey Hospital, Canada Avenue, Redhill, Surrey, RH1 5RH, U.K.

${ }^{1}$ Acute Stroke Unit, East Surrey Hospital, Canada Avenue, Redhill, Surrey, RH1 5RH, U.K. vpotter@doctors.org.uk
The chronic form of heel pain is now more commonly termed 'plantar fasciosis'. Repetitive trauma and overuse leads to microtears, with cycles of tearing and healing causing the release of various chemical mediators. This is thought to lead to myxoid degeneration and weakness of the fascia as well as pain. $^{2}$ This is a degenerative fasciosis without inflammation. ${ }^{3}$

Plantar fasciitis can also be associated with various inflammatory systemic conditions such as gout and rheumatoid arthritis. An increased incidence exists in patients with certain HLA B27 sponyloarthropathies in which case the fasciitis often presents at a younger age and carries a poorer prognosis. ${ }^{4}$ 


\section{Differential Diagnosis for Plantar Fasciitis \\ Foreign body \\ Infection \\ Tumors or metastases of the foot's plantar aspect \\ Peripheral neuropathy \\ Calcaneal stress fracture \\ Plantar fascia rupture \\ Bruised heel syndrome \\ Sciatica \\ Tarsal tunnel syndrome \\ Entrapment of the lateral plantar nerve \\ Subcalcaneal bursitis \\ Calcaneal apophysitis \\ Systemic inflammatory conditions \\ Gout \\ Osteomyelitis \\ Pagets Disease \\ Diabetic fascial disease \\ Plantar xanthoma \\ Plantar vein thrombosis}

Table 1 Differential diagnosis for plantar fasciitis.

\section{Clinical features}

Typically the patient presents with pain in one or both plantar aspects of the feet. This has usually been insidious in onset although occasionally can be acute. The pain may be poorly localized particularly initially, but then may become maximal at the origin of the fascia, to the medial calcaneal tuberosity. It tends to be worse in the morning when the foot is first put on the floor or following a period of rest and is relieved on movement during the day. However, it can again be exacerbated by increased weight-bearing activity such as running. It is often described as a 'tearing' pain. On inspection, the foot appears normal with either no or slight swelling. ${ }^{5}$ Palpating the foot, the practitioner may elicit maximal tenderness at the aforementioned origin of the plantar fascia and pressure here reproduces the pain. Passive dorsiflexion of the toes and ankles can also cause pain by stretching the fascia. ${ }^{6}$ The clinician must also consider a differential diagnosis for heel pain. (Table 1)

\section{Investigations in plantar fasciitis}

The diagnosis of plantar fasciitis is a clinical one based on the history and examination. It is also usually self-limiting and managed conservatively. However, in particular circumstances such as atypical presentations or failing to respond to treatment, the clinician may wish to consider other tests to aid in the diagnosis and differential diagnosis of heel pain syndrome.

\section{Blood tests}

In most cases, these do not play a role in diagnosis although occasionally the clinician may wish to perform certain blood tests if considering a systemic condition or alternative cause. A raised erythrocyte sedimentation rate or C-reactive protein may indicate underlying inflammation or infection respectively. Likewise, testing for HLA B27 gene may be helpful if suspecting one of the HLA B27 spondyloarthropathies such as ankylosing spondylitis, psoriatic arthritis, reactive arthritis or enteropathic arthritis. Further examples include gout in which serum uric acid is usually raised and a diagnosis of Paget's disease is supported by a raised alkaline phosphatase with a normal phosphate and calcium.

\section{Plain radiograph}

It is common for a plain lateral radiograph of the foot to be ordered, often to rule out a stress fracture, heel spurs, fractured spurs or some other bony cause of the patient's heel pain. However, one study involving 215 heels concluded that routine radiographs are of limited use in the initial evaluation of adults presenting with non-traumatic heel pain and should be reserved for those who do not improve or have an unusual history or physical signs. This study also found that radiographs were normal in $17.2 \%$ and that incidental radiographic findings were observed in $81.4 \%$; most commonly plantar calcaneal spurs and Achilles spurs $(46.5 \%, 100$ of 215$){ }^{7}$ 
One may argue that these incidental findings could in some cases explain the patient's symptoms although a heel spur does not have any diagnostic value. ${ }^{8}$ The aforementioned study also found that only $2 \%$ of the patients in their study had abnormal findings that prompted further evaluation supporting the conclusion that radiographs are generally of limited use and hence not cost effective. ${ }^{7}$

\section{Ultrasound}

In difficult cases, the clinician may wish to request an ultrasound of the foot. A number of studies have sought to evaluate usefulness of this modality with respect to diagnosing plantar fasciitis. One such study found that in patients with plantar fasciitis, ultrasound may detect relatively small differences in plantar fascia thickness $(2.9 \mathrm{~mm}$ in patients with unilateral plantar fasciitis, $2.2 \mathrm{~mm}$ for the contralateral heel and $2.5 \mathrm{~mm}$ for the control group). ${ }^{9}$ A similar conclusion was reached by a Cardinal, et al., who found that increased thickness of the fascia and hypoechoic fascia are sonographic findings of this condition. They concluded that ultrasound may be a valuable noninvasive technique for the diagnosis of plantar fasciitis. $^{10}$

\section{Bone Scintigraphy}

Bone scintigraphy is an investigation which uses technetium-99m labelled diphosphonates. It is one of the most frequently performed of all radionuclide procedures. The isotope is injected into the patient intravenously and is then cleared from the blood and taken up by the skeleton. Blood flow and osteoblastic activity determine skeletal uptake. This mode of imaging is not specific but is very sensitive and so is useful in screening for many pathological conditions. It is often used to investigate potential malignant bony lesions but may be requested, as in the context of heel pain, to explore chronic foot pain which is not responding as expected. In cases of musculoskeletal trauma, radionuclide bone imaging is useful for identifying pathologic conditions for which radiographs may be non-diagnostic. ${ }^{11}$ It is often used to rule out a stress fracture.
Several studies have sought to determine the value of this modality in the diagnosis of plantar fasciitis. One study compared ultrasonography and bone scintigraphy; both were found to be sensitive and specific diagnostic imaging investigations in this condition. This study found that both confirmed the clinical diagnosis in 25 of 27 heels. However, this again serves to highlight the accuracy of clinical diagnosis supporting the view that imaging is only rarely required. Comparing the two investigations, the differences in sensitivity and specificity were not statistically significant and given the relative ease and non invasiveness of ultrasonography, this would thus seem to be the choice of imaging to employ. ${ }^{12}$ Another study found that focal uptake at the medial calcaneal tubercle was present in the majority of patients in their study who had been diagnosed with plantar fasciitis, thus confirming the diagnosis. ${ }^{13} \mathrm{~A}$ third study came to the conclusion that scintigraphy is very useful in diagnosing plantar fasciitis and distinguishing it from other causes of a painful heel after imaging 15 patients with chronic heel pain; 10 demonstrated scan findings consistent with plantar fasciitis whilst 2 were found to have a calcaneal stress fracture. ${ }^{14}$

\section{Magnetic resonance imaging}

Magnetic resonance imaging (MRI) is a further possible investigation to perform in difficult cases and is useful in detecting tears or ruptures of the fascia. In plantar fasciitis the MRI may show a widened fascia with increased signal often with some reactive oedema in the adjac ent bone. ${ }^{15}$ The normal thickness of the plantar fascia is $4 \mathrm{~mm}$; in plantar fasciitis this may be increased to $8 \mathrm{~mm}$. In cases of ruptures and tears this thickness can reach $10 \mathrm{~mm}$ or more and additionally, the MRI will show intrafascial high signal intensity of the T2 weighted image. ${ }^{16}$ One study found that maximal thickness of the plantar fascia was significantly increased in patients with plantar fasciitis and that feet with the condition had areas of moderately increased signal intensity in the substance of the fascia. ${ }^{17}$ 
Although therefore very useful, a study compared the diagnostic accuracy of ultrasound and MRI and found the two comparable and concluded MRI may be reserved for the more complex cases where the diagnosis is not clear. ${ }^{18}$ Similarly, another paper found that although MRI is the modality of choice in the morphologic assessment of different plantar fascia lesions, sonography can also serve as an effective tool and may substitute MRI in the diagnosis of plantar fasciitis. ${ }^{19}$

\section{Nerve conduction studies}

This is used to look at the ability of electrical conduction of a nerve and so to diagnose nerve damage or dysfunction. It involves the placement of electrodes on the skin at intervals along the nerve in question; a low intensity electrical current is then applied to generate an impulse. Nerve conduction velocity can then be measured by recording the motor response of a muscle to the stimulation of its motor nerve. Nerve conduction studies are rarely required in the context of plantar fasciitis, but may be considered if the clinician is suspecting nerve entrapment as the cause of the patient's pain. This may include tarsal tunnel syndrome or where an atypical presentation represents a mixed picture. For example, Chang, et al., found that sensory nerve conduction studies were a useful and objective tool in the diagnosis of medical calcaneal neuropathy and that there was an association between this neuropathy and plantar fasciitis. ${ }^{20}$ In addition, Baxter's neuropathy, which is referred to as entrapment of the first branch of the lateral plantar nerve can produce medial heel pain. This can be very similar to that caused by plantar fasciitis and is thought to account for up to $20 \%$ of heel pain. ${ }^{21}$

\section{Conclusion}

Plantar fasciitis is a common cause of heel and foot pain. In most cases, history and examination provide the diagnosis and treatment can be advised based on severity and duration of symptoms.
If there is doubt as to the cause, investigations, usually rarely indicated, can be considered. Imaging modalities and tests include $\mathrm{x}$-rays, ultrasound, scintigraphy, MRI and nerve conduction studies. The main value of these are in confirming the clinical diagnosis of plantar fasciitis as diagnostic features on imaging do exist and in detecting other causes of the patient's heel pain.' Plain radiography is generally unhelpful; ultrasound appears to be the most useful in difficult cases and has the additional advantages of speed and low cost. MRI, bone scintigraphy and nerve conduction studies are best reserved for when there is a strong clinical suspicious of an alternative diagnosis or when the patient's heel pain is not responding after 3 months or more of treatment. ${ }^{6}$

\section{References}

1. Puttaswamaiah R, Chandran P: Degenerative plantar fasciitis: A review of current concepts. The Foot 17: 3 - 9, 2007.

2. Tsai WC, Wang CL, Tang FT, Hsu TC, Hsu KH, Wong MK: Treatment of proximal plantar fasciitis with ultrasound-guided steroid injection. Arch Phys Med Rehabil 81: 1416 - 1421, 2000. 3. Lemont $\mathrm{H}$, Ammirati KM, Usen N: A degenerative process (fasciosis) without inflammation. J American Podiatric Medical Association 93: 234 - 237, 2003.

4. Foye PM, Stitik TP: Plantar fasciitis. Emedicine, 2008. Accessed 09/23/2009.

5. Amis J, Jennings L, Graham D, Graham CE: Painful heel syndrome: radiographic and treatment assessment. Foot Ankle 9: $91-95,1988$.

6. Duff G: Plantar fasciitis and heel pain. Reports on the rheumatic diseases 2: 1 - 4, 2004.

7. Levy JC, Mizel MS, Clifford PD, Temple HT:Value of radiographs in the initial evaluation of nontraumatic adult heel pain. Foot Ankle Int 27: 427 - 430, 2006.

8. Tanz SS: Heel pain. Clin Orthop 28: 169 - 178, 1963. 9. Ozdemira H, Yilmazb E, Murata A, Karakurtb L, Poyraza AK, Ogura E: Sonographic evaluation of plantar fasciitis and relation to body mass index. Eur J Radiology 54: 443 - 447, 2005.

10. Cardinal E, Chhem RK, Beauregard, CG, Aubin B, Pelletier, M: Plantar fasciitis: sonographic evaluation. Radiology 201: 257 259, 1996.

11. Love C, Din AS, Tomas MB, Kalapparambath TP, Palestro CJ: Radionuclide bone imaging: An illustrative review. Radiographics 23 (2): 341 - 358, 2003.

12. Kane D, Greaney T, Shanahan M, Duffy G, Bresnihan R, Fitzgerald $\mathrm{O}$ : The role of ultrasonogaphy in the diagnosis and management of idiopathic plantar fasciitis. Rheumatology 40: 1002 - 1008, 2001. 
13. O’Duffy EK, Clunie GP, Gacinovic S, Edwards JC, Bomanii JB, Ell PJ: Foot pain: specific indications for scintigraphy. Br J Rheumatol 37: 442 - 447, 1998.

14. Intenzo CM, Wapner KL, Park CH, Kim SM: Evaluation of plantar fasciitis by three-phase bone scintigraphy. Clin Nucl Med 16: 325 - 328, 1991.

15. Ostlere S: Imaging the ankle and foot. Imaging 2003;15: 242 $-269$.

16. Kline A: Plantar fascial rupture of the foot: a case report. The Foot and Ankle Online Journal 2 (5): 4, 2009.

17. Berkowtiz JF, Kier R, Rudicel S: Plantar fasciitis: MR imaging. Radiology 179: 665 - 667, 1991.

18. Abdel-Wahab N, Fathi S, Al-Emadi S, Mahdi S: High resolution ultrasonographic diagnosis of plantar fasciitis: a correlation of ultrasound and magnetic resonance imaging. International Journal of Rheumatic Disease 11: 279 - 286, 2008. 19. Sabir N, Demirlenk S, Yagci B, Karabutlut N, Cubukcu S: Clinical utility of sonography in diagnosing plantar fasciitis. J Ultrasound Med 24: 1041 - 1048, 2005.

20. Chang CW, Wng YC, Hou WH, Lee XX, Chang KF: Medial calcaneal neuropathy is associated with plantar fasciitis. The Journal of Foot and Ankle Surgery 118: 119 - 123, 2007.

21. Chundru U, Liebeskind A, Seidelmann F, Fogel J, Franklin P, Beltran J: Plantar fasciitis and calcaneal spur formation are associated with abductor digiti minimi atrophy on MRI of the foot. Skeletal Radiology 37: 505 - 510, 2008. 